Results: Findings included: a) the volunteers who received training stated an increase in their confidence to respond to emergencies, b) a $65 \%$ increase of Red Cross volunteers, c) a increased awareness of EMS within the province, d) greater community engagement at emergency scenes, and e) broad respect from the community towards the HDRC volunteers.

Discussion: Considerations for the future include: a) development of a continuing education program, b) increase of supplies to volunteers, c) more training involving multi casualty incidents, d) development of a communications protocol between volunteers and other healthcare providers and e) limit CPR training to drowning related events.

Conclusion: The outcomes exceeded the planned goals: knowledge and retention of course materials and skills is good, confidence levels of volunteers increased and those that are involved in emergency events in Hai Duong province are safer. The profile of EMS and first responders as a critical component of community health has been measurably raised among key stakeholders and the community.

Prehosp Disaster Med 2011;26(Suppl. 1):s95-s96

doi:10.1017/S1049023X11003013

(A285) Planning and Organization of Emergency Medical Services in Mumbai

A. Prakash, R. Nagose

Jamsetji Tata Centre for Disaster Management, Mumbai, India

In the past two decades, Mumbai has witnessed several masscasualty incidents. Somehow, it seems that the city has missed some important lessons from these events. Mumbai has no formal structure for emergency medical services (EMS). Although EMS may seem to be a much-desired necessity, scholars have raised questions on the practicality and feasibility of having such a system in Mumbai. Factors such as population congestion, traffic volume, and lack of coordination among existing hospitals, the success of such a system in a city like Mumbai is jeopardized. In spite of having similar challenges in some other regions of the country, EMS systems (e.g., in Gujarat) have achieved substantial success. This paper deals with the planning and organization of EMS in Mumbai. It evaluates the performances of the existing EMS systems in other Indian cities. The paper also discusses the advantages of having such a system, particularly during the events such as disasters, accidents, acts of terrorism, etc. The paper also discusses the possible consequences of the absence of EMS, such as delayed ambulance dispatch, improper distribution of patients, overcrowding at certain hospitals thereby leading to poor triage, and several similar problems that can worsen a crisis. It studies the potential challenges for the establishment of such a system in Mumbai, and suggests a model for an effective EMS system for the city. Prehosp Disaster Med 2011;26(Suppl. 1):s96 doi:10.1017/S1049023X11003025

(A286) Using a Computer Simulation (CS) to Improve Training and Event Management of Paramedics for Mass Casualty Incidents (MCI)

E. Jaffe, ${ }^{1}$ A. Dagan, ${ }^{2}$ E. Zahavi ${ }^{1}$

1. Emergency Medicine, Beer Sheva, Israel

2. Emergency Medicine, Jerusalem, Israel
Using a Computer Simulation (CS) to improve training and event management of paramedics for Mass Casualty Incidents (MCI). Eli Jaffe, Avi Dagan, Eyal Zahavi, Einat Aviel, Bruria Adini. The Department of Emergency Medicine, Ben-Gurion University of the Negev Magen David Adom (MDA) is the national emergency organization in Israel. Over the past few decades MDA personnel have been required to deal with MCIs involving large numbers of casualties. Recently, there have been fewer terrorist related MCIs, however, there is a continuing need for to maintain the knowledge and skills of paramedics to manage MCIs.

Objective: To examine performance of paramedics exposed to a CS compared to a control group exposed to a traditional lecture based learning experience.

Method: An interactive CS based on the MDA standard operating procedure for managing MCIs was developed. The participants were randomly divided into two groups. Group 1 received the lecture format, and Group 2 the CS. Both groups were given a pre-test (Group 1 average score 56.3, Group 2 average score 53.1), and two post-tests. One immediately following completion of the intervention, and a second a month after completion of the course.

Results: Average scores for the CS Group $(n=15)$ was significantly different on the first post-test (Group 1 average score 53.2, Group 2 average score 68.7), by 30\% and on the second (Group 1 average score 71.9 , Group 2 average score 80.8$)$ by $12 \%$ compared to the Lecture Group $(N=17)(P=<0.00)$.

Conclusion: CS allow for the use of multiple media formats based on real events, and are able to replicate reality using real media material. MDA has recommended that continuing education interventions for emergency medical personnel for maintaining knowledge and skills required for the management of MCIs utilize a CS based training methods.

Prehosp Disaster Med 2011;26(Suppl. 1):s96 doi:10.1017/S1049023X11003037

\section{(A287) Animal Health in Disasters: Why does it Matter? P. Hollier}

Veterinary Stability Operations, Tucker, United States of America

Animal production is an important component to economic stability and food security in developing country. Economic development programs have targeted agriculture in developing country as a means of decreasing poverty and empowering vulnerable populations throughout much of the developing world. Disasters due to natural and man-made hazards that impact animal agriculture have a disproportionate impact on the rural poor and vulnerable populations affecting the economic well being and health of the greater population. Protection of livelihoods dependent on animal agriculture is important to consider in community resilience and humanitarian assistance activities that range from capacity building through response and recovery. In order to meet the challenges of the next generation of global health threats, policy, research, and practice must adopt a new cross-cutting approach that targets the human-animalecologic interface taking into account political, socioeconomic, and cultural factors.

Prehosp Disaster Med 2011;26(Suppl. 1):s96 doi:10.1017/S1049023X11003049 